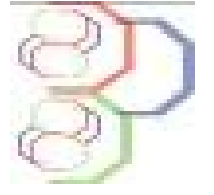

Journal of Applied Biosciences 66:5049 - 5059

\title{
Perceptions locales des déterminants de la fragmentation des îlots de forêts denses dans la région des Monts Kouffé au Bénin
}

\author{
Inoussa Toko Mouhamadou ${ }^{1 *}$, Ismaila Toko Imorou², Aminou Sakari Mèdaho³ \& Brice Sinsin 4 \\ ${ }^{1 *}$ Département de SIG. RECTAS. Obafemi Awolowo University Campus. Off Road1, PMB : 5545, lle-Ife. Osun State, \\ NIGERIA. \\ 2Laboratoire de Cartographie. Département de Géographie et Aménagement du Territoire. Faculté des Lettres, Arts \\ et Sciences Humaines. Université d'Abomey-Calavi. 01 BP 526 Cotonou. Bénin. \\ ${ }^{3}$ Collège d'Enseignement Général 1 de Bantè. BP 05, Bantè. Bénin. \\ 4Laboratoire d'Ecologie Appliquée. Département Aménagement et Gestion de l'Environnement. Faculté des \\ Sciences Agronomiques. Université d'Abomey-Calavi. 01 BP 526 Cotonou. Bénin. \\ Auteur correspondant, emails : tinoussa@hotmail.com / toko@rectas.org \\ Original submitted in on $8^{\text {th }}$ February 2013 Published online at www.m.elewa.org on 30 th June 2013. \\ https://dx.doi.org/10.4314/iab.v66i0.95002
}

\section{RÉSUMÉ}

Objectif : Cette étude a pour but d'évaluer les facteurs naturels et anthropiques sources de perturbations des écosystèmes forestiers dans la région des Monts des Kouffé en vue d'une planification de leur gestion.

Méthodologie et résultats: Les méthodes utilisées sont le focus group, l'interview et le modèle PEIR (Pression/Etat/Impact/Réponse). Celles-ci ont permis de comprendre que les groupes socioculturels fon et Bariba estiment que l'érosion hydrique est le principal facteur de la fragmentation des forêts denses. Tandis que pour les Lokpa, et les Peulh, la fragmentation des forêts denses a pour cause la pauvreté des sols. De même, les groupes socioculturels estiment que la durée de la fragmentation des forêts denses est de 1 et 5 ans ; 5 et 10 ans pour certains et de 10 ans et plus pour d'autres. Pour les catégories socioprofessionnelles, cette durée est de 1 à 5 ans et de 5 à 10 ans seulement.

Conclusions et applications des résultats : Dans la région des Monts Kouffé, l'exploitation forestière, les feux de végétation, l'agriculture, l'érosion hydrique et la pauvreté des sols sont considérés comme les facteurs déterminants de la fragmentation des forêts denses. Ces résultats ainsi obtenus permettront aux ONG du domaine de la conservation des ressources naturelles d'alerter les décideurs politiques surtout les Députés de prendre des lois à l'Assemblée Nationale en vue de corriger cette situation.

Mots clés : Perceptions locales, facteurs déterminants, dégradation des forêts denses, Région des Monts Kouffé. 
Local perceptions of the determinants of the degradation of the dense forests of the small islands in the area of the Kouffé Mounts in Benin

\begin{abstract}
Objective: The aim of this study is to evaluate the natural and anthropic factors sources of disturbances of the forest ecosystems in Monts Kouffé zone for a planning of their management.

Methodology and results: The methods used were focus group, interviews, and the PEIR (Pression/Etat/Impact/Réponse) model. Those that understand the Fon and Bariba sociocultural groups estimate that hydrolic erosion is the major factor of dense forests destruction. While for Lokpa, and Peulhs dense forests disintegration is because of poor soils. In the same way, the sociocultural groups estimate that the duration of dense forests fragmentation is 1 to 5 years; 5 to 10 years for some, 10 years and more for others. For the socio-professional categories this duration is from 1 to 5 years and 5 to 10 years only.

Conclusions and application: In Monts Kouffé zone, the wood exploitation, vegetation fires, agriculture, hydraulic erosion, and poor soils are regarded as main factors of dense forests disintegration. These obtained results will allow NGOs in the natural resources conservation field to alert decision makers especially Members of parliament to take rules at National Assembly in order to mitigate this forest ecosystem degradation.

Keywords: Local perceptions, main factors, dense forests degradation, Monts Kouffé zone.
\end{abstract}

\section{INTRODUCTION}

Les forêts tropicales sont des écosystèmes extrêmement utiles et précieux pour l'humanité (Tchatal, 1999). Elles jouent un rôle capital dans la régulation de l'effet de serre, dans les grands équilibres climatiques, dans la satisfaction de nombreux besoins des populations locales et constituent le grand réservoir de la biodiversité de la planète (FAO, 1999). L'éclosion de la biodiversité constitue de nos jours une menace pour l'humanité. La question qui se pose est de savoir comment sauver la biodiversité très menacée. Selon Gaffan (2001), la dégradation du milieu naturel avec les ressources et la fragilité des écosystèmes tropicaux évoluent à un rythme alarmant ces dernières années. Les phénomènes de dégradation et de fragmentation des ressources naturelles constituent une menace pour l'humanité et est devenu depuis la conférence de Rio en 1992, une préoccupation fondamentale. Depuis lors, la communauté scientifique d'évaluation des écosystèmes pour le millénaire affirme que la fragmentation écologique est devenue une des premières causes d'atteinte à la biodiversité avant la pollution. La fragmentation des forêts modifie la taille, la forme et l'isolement du paysage générant ainsi un impact sur les fonctions écopaysagères des éléments (Wiens, 1997). En Afrique de l'Ouest, les formes dominantes de perturbation qui contribuent à façonner la structure et la physionomie de la végétation au sein des aires protégées sont les feux de végétation et l'irrégularité de la pluviométrie (Hien et Al., 2002). Pour Sinsin (1996), les feux de végétation sont largement pratiqués et sont les facteurs déterminants dans l'évolution régressive de toute une série de paysages végétaux. Au Bénin; la dégradation du couvert végétal s'accompagne d'une baisse de fertilité des sols entrainant des déplacements de population vers des zones plus favorables. La dégradation de la végétation est en partie due au défrichement lié à la culture itinérante sur brulis. Pour satisfaire leurs besoins, les populations vont à la recherche de terres fertiles (Mercier, 1991). II est donc clair que pour comprendre la genèse des îlots de forêts denses de la région des Monts Kouffé il faut nécessairement connaître les facteurs déterminants et la durée de dégradation de ces forêts denses. 


\section{MATERIELS ET METHODES}

Matériels: Les matériels utilisés dans cette étude concernent un questionnaire d'enquête de terrain et des documents obtenus dans les bibliothèques et centres de documentation et sur l'internet.

\section{Démarche méthodologique}

Échantillonnage: Les individus enquêtés sont constitués de chasseurs, de paysans, de scieurs et d'exploitants forestiers. La taille de l'echantillon est de 100 individus choisis au hasard.

Collecte des données socio-économiques: Les méthodes utilisées sont le focus group, l'interview et le modèle PEIR (Pression/Etat/

Impact/Réponse).

Focus Group Discussion : Le Focus Group Discussion ou Discussion du Groupe Focalisé encore appelé animation de groupe est un groupe de discussion qui rassemble des personnes de même milieu, du même sexe ou ayant des expériences ou âges semblables pour discuter d'un thème précis présentant un intérêt pour le chercheur. De façon plus explicite, le Focus Group Discussion est une technique d'entretien qui réunit six à douze participants dans le cadre d'une discussion structurée autour d'un sujet particulier (Azalou, 2008). Ainsi deux focus group composés respectivement de chasseurs, de paysans, de scieurs et d'exploitants forestiers sont réalisés au niveau de chacun des six villages du secteur d'étude. La taille du focus group varie entre 8 et 10 par village. Ces villages ont été choisis en tenant compte de leur répartition spatiale dans le secteur

\section{RESULTATS}

Perception des groupes socioculturels

Facteurs de fragmentation des forêts denses selon les groupes socioculturels : La figure 1 présente sur deux axes, les perceptions des facteurs déterminants de la fragmentation. Dans cette figure 1, les fon et les Bariba d'étude et surtout de leur proximité vis-à-vis de la forêt classée et des principaux groupes socioculturels qui les composent. Cette méthode a permis de collecter des informations permettant d'évaluer les effets de la fragmentation sur les espèces exploitées dans la forêt classée, les raisons qui motivent le choix des espèces forestières utilisées afin de proposer des activités et mesures moins destructives des ressources végétales et animales de ladite forêt classée.

Interview avec les différents acteurs de la forêt classée: Une interview est accordée aux différents acteurs de la forêt classée plus précisément aux chefs chasseurs, exploitants forestiers, éleveurs et paysans du secteur d'étude. Celle-ci a permis d'avoir des informations sur les espèces végétales et animales disparues, celles en voie de disparition ainsi que les effets liés au phénomène de la fragmentation.

Utilisation du modèle PEIR : Le modèle PEIR est une approche utilisée ici pour connaître l'ensemble des facteurs anthropiques liés au phénomène de la fragmentation des habitats naturels.

Traitement des données :

Les données d'enquête ont été traitées avec le logiciel Mini Tab qui nous a permis de réaliser des analyses factorielles de correspondances sur les différentes perceptions des populations locales. A partir de ces analyses divers facteurs responsables de la fragmentation des îlots de forêts denses sont connus.

estiment que l'érosion hydrique est le principal facteur de la fragmentation des forêts denses. Pour les Lokpa et les Peulh, la fragmentation des forêts denses a pour cause la pauvreté des sols. 


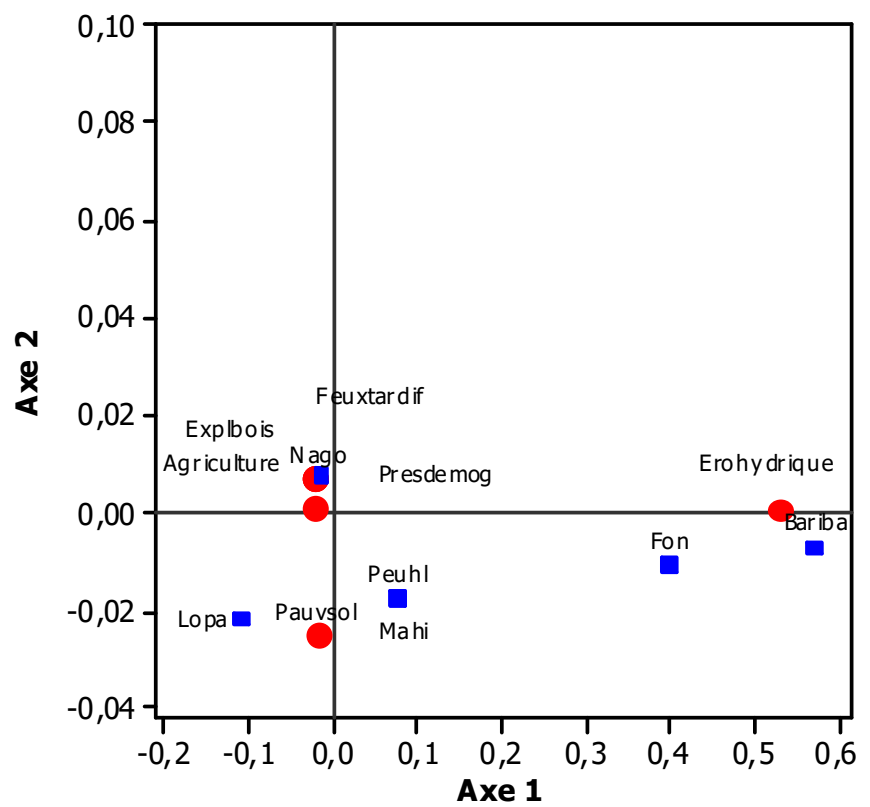

Figure 1 : Projection des perceptions des facteurs de fragmentation des forêts denses et des différents groupes socioculturels dans un système d'axes

Ordre d'importance des facteurs déterminants de la fragmentation des forêts denses selon les groupes socioculturels : Le tableau1 présente selon les groupes socioculturels l'ordre d'importance des facteurs déterminants de la fragmentation.

Tableau 1 : Importance des axes de l'analyse factorielle de correspondance (AFC) entre les facteurs de fragmentation des forêts denses et les groupes socioculturels

\begin{tabular}{|c|c|c|c|c|}
\hline Axe & Inertia & Proportion & Cumulative & Histogram me \\
\hline 1 & 0,0135 & 0,9894 & 0,9894 & 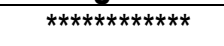 \\
\hline 2 & 0,0001 & 0,0106 & 1,0000 & \\
\hline Total & 0,0136 & & & \\
\hline
\end{tabular}

Les résultats de l'analyse factorielle de correspondance (AFC) entre les facteurs de fragmentation des forêts denses et les groupes socioculturels révèlent que les deux premiers axes expliquent $98,94 \%$ des informations obtenues (Tableau 1), ce qui est suffisant pour faire une bonne synthèse des informations.

Durée de la fragmentation: Cette importance de la durée de fragmentation selon les groupes socioculturels est exprimée ici dans le tableau 2.

Tableau 2 : Importance des axes de l'analyse factorielle de correspondance (AFC) entre les perceptions de la durée de fragmentation des forêts denses et les groupes socioculturels.

\begin{tabular}{|c|c|c|c|c|}
\hline Axe & Inertia & Proportion & Cumulative & Histogram me \\
\hline 1 & 0,0925 & 0,6628 & 0,6628 & $* \star * \star * \star * \star * \star *$ \\
\hline 2 & 0,0471 & 0,3372 & 1,0000 & $* * * * * *$ \\
\hline Total & 0,1395 & & & \\
\hline
\end{tabular}

Les résultats de l'analyse factorielle de correspondance (AFC) entre les perceptions de la durée de fragmentation des forêts denses et les groupes socioculturels révèlent que les deux premiers axes expliquent $100 \%$ des informations obtenues (Tableau 2), ce qui est suffisant pour faire une bonne synthèse des informations. La figure 2 présente sur deux axes, les perceptions de la durée de fragmentation pour ces groupes socioculturels. 


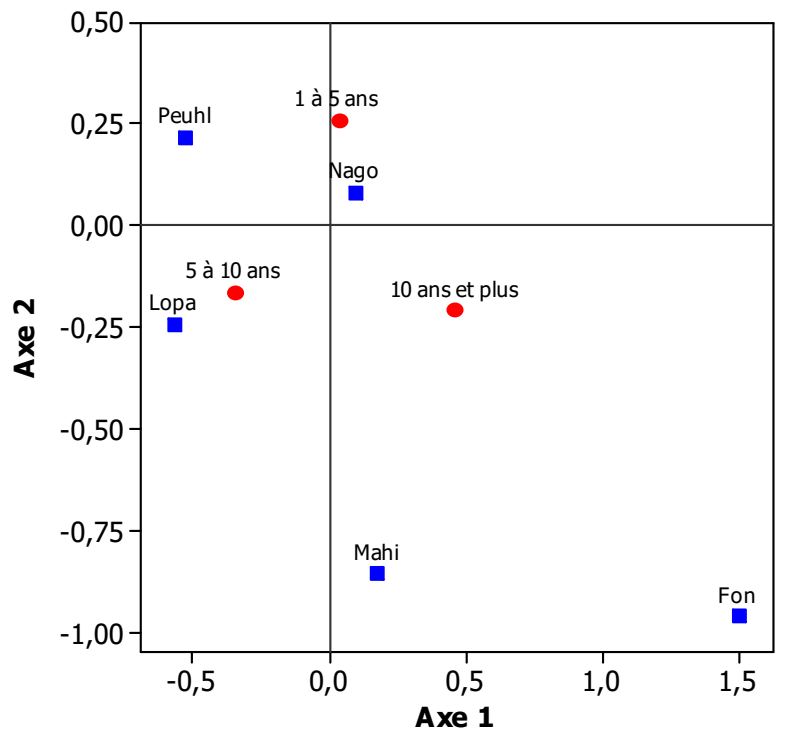

Figure 2 : Projection des perceptions de la durée de fragmentation des forêts denses et des différents groupes socioculturels dans un système d'axes

Sur l'axe 1 de la figure2, les Fon estiment que la durée de la fragmentation des forêts denses est de 10 ans et plus alors que les Peulh et les Lokpa pensent que cette durée est comprise entre 5 et 10 ans. Sur l'axe 2, les Mahi pensent comme les Fon que la durée de la fragmentation des forêts denses est de 10 ans et plus alors que les Nago l'estiment entre 1 et 5 ans.
Perception des catégories socioprofessionnelles Facteurs de fragmentation des forêts denses selon les catégories socioprofessionnelles : Le Tableau 3 présente l'importance des axes de l'analyse factorielle de correspondance (AFC) entre les facteurs de fragmentation des forêts denses et les catégories socioprofessionnelles.

Tableau 3 : Importance des axes de l'analyse factorielle de correspondance (AFC) entre les facteurs de fragmentation des forêts denses et les catégories socioprofessionnelles

\begin{tabular}{|c|c|c|c|c|}
\hline Axe & Inertia & Proportion & Cumulative & Histogram me \\
\hline 1 & 0,0025 & 0,8918 & 0,8918 & $* \star * \star \star \star \star \star \star \star * *$ \\
\hline 2 & 0,0003 & 0,0997 & 0,9915 & $* \star *$ \\
\hline 3 & 0,0000 & 0,0085 & 1,0000 & \\
\hline Total & 0,0027 & & & \\
\hline
\end{tabular}

Les résultats de l'analyse factorielle de correspondance (AFC) entre les facteurs de fragmentation des forêts denses et les catégories socioprofessionnelles révèlent que les deux premiers axes expliquent $99,15 \%$ des informations obtenues (Tableau 3), ce qui est suffisant pour faire une bonne synthèse des informations. Comme dans l'autre cas, la figure 3 présente sur deux axes ici également, les perceptions des facteurs de fragmentation pour les groupes socioprofessionnels. 


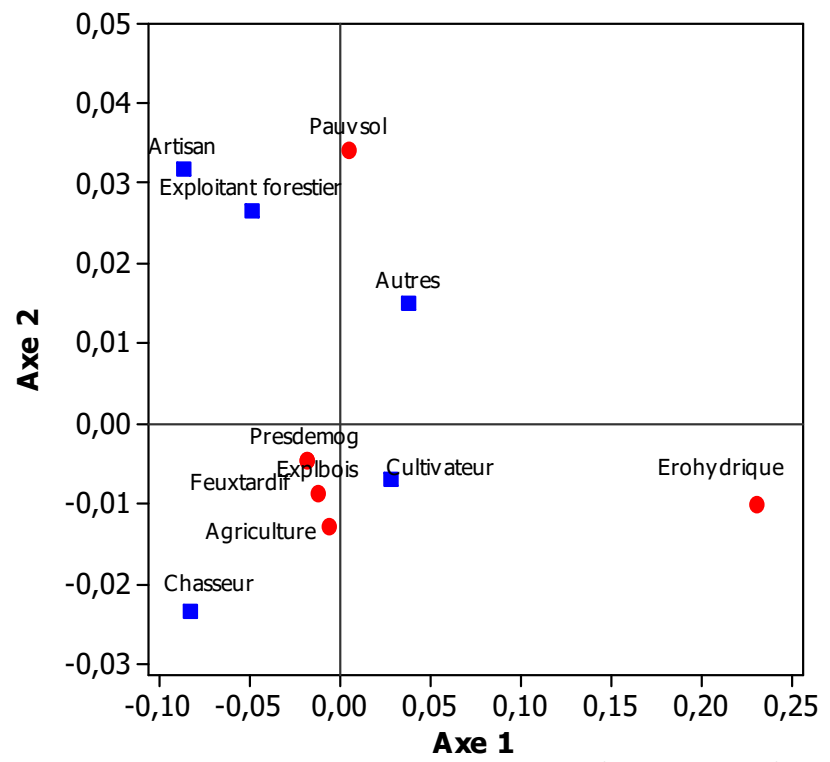

Figure 3: Projection des perceptions des facteurs de fragmentation des forêts denses et des différents groupes socioprofessionnels dans un système d'axes

Sur l'axe 1, les cultivateurs estiment que l'érosion hydrique est le principal déterminant de la fragmentation des forêts denses alors que pour les chasseurs et les artisans, c'est plutôt les feux tardifs. Par ailleurs, sur l'axe 2 , les exploitants forestiers estiment que la pauvreté des sols est le principal déterminant de la fragmentation des forêts denses.
Ordre d'importance des facteurs déterminants de la fragmentation des forêts denses selon les catégories socioprofessionnelles: Cet ordre d'importance des facteurs déterminants de la fragmentation selon les catégories socioprofessionnelles est présenté par le tableau 4.

Tableau 4 : Importance des axes de l'analyse factorielle de correspondance (AFC) entre les facteurs de fragmentation des forêts denses et les catégories socioprofessionnelles

\begin{tabular}{|c|c|c|c|c|}
\hline Axe & Inertia & Proportion & Cumulative & Histogram me \\
\hline 1 & 0,1325 & 0,4556 & 0,4556 & 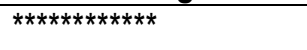 \\
\hline 2 & 0,0976 & 0,3354 & 0,7910 & 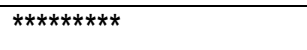 \\
\hline 3 & 0,0441 & 0,1517 & 0,9427 & $\overline{k \star \star \star \star \star \star \star ~}$ \\
\hline 4 & 0,0167 & 0,0573 & 1,0000 & $* * *$ \\
\hline Total & 0,2909 & & & \\
\hline
\end{tabular}

Les résultats de l'analyse factorielle de correspondance (AFC) entre les facteurs de fragmentation des forêts denses et les catégories socioprofessionnelles révèlent que les deux premiers axes expliquent $79,10 \%$ des informations obtenues (Tableau 4), ce qui est suffisant pour faire une bonne synthèse des informations. La figure 4 présente sur deux axes les perceptions de la durée de fragmentation des forêts denses et les différents groupes socioprofessionnels. 


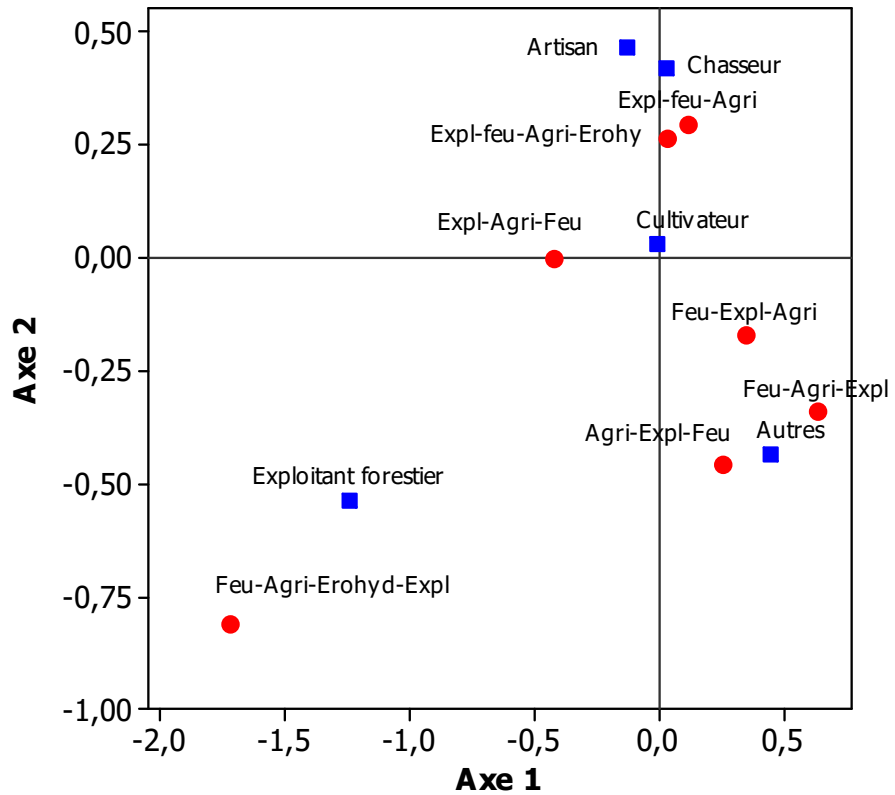

Figure 4: Projection des perceptions de facteurs de fragmentation des forêts denses et des différents groupes socioprofessionnels dans un système d'axes

Sur l'axe 1, les exploitants forestiers estiment que l'exploitation forestière suivie de l'agriculture et des feux tardifs de végétation est les principales causes de la fragmentation des forêts denses alors que pour les autres acteurs, c'est plutôt les feux tardifs suivie de l'agriculture et de l'exploitation forestière. Par ailleurs, sur l'axe 2, les chasseurs pensent que l'exploitation forestière suivie des feux tardifs, de l'agriculture et de l'érosion hydrique est les principaux déterminants de la fragmentation des forêts denses.
Durée de la fragmentation : Les résultats de l'analyse factorielle de correspondance (AFC) entre les perceptions de la durée de fragmentation des forêts denses et les catégories socioprofessionnelles révèlent que les deux premiers axes expliquent $100 \%$ des informations obtenues (Tableau 5), ce qui est suffisant pour faire une bonne synthèse des informations. Dans la figure 5 , sur deux axes sont présentés les perceptions de la durée de fragmentation des forêts denses et les différents groupes socioprofessionnels.

Tableau 5 : Importance des axes de l'analyse factorielle de correspondance (AFC) entre les perceptions de la durée de fragmentation des forêts denses et les catégories socioprofessionnelles

\begin{tabular}{|c|c|c|c|l|}
\hline Axe & Inertia & Proportion & Cumulative & \multicolumn{1}{|c|}{ Histogram me } \\
\hline 1 & 0,1611 & 0,8236 & 0,8236 & $* * \star \star * \star * \star * \star * *$ \\
\hline 2 & 0,0345 & 0,1764 & 1,0000 & $* * * * *$ \\
\hline Total & 0,1956 & & & \\
\hline
\end{tabular}




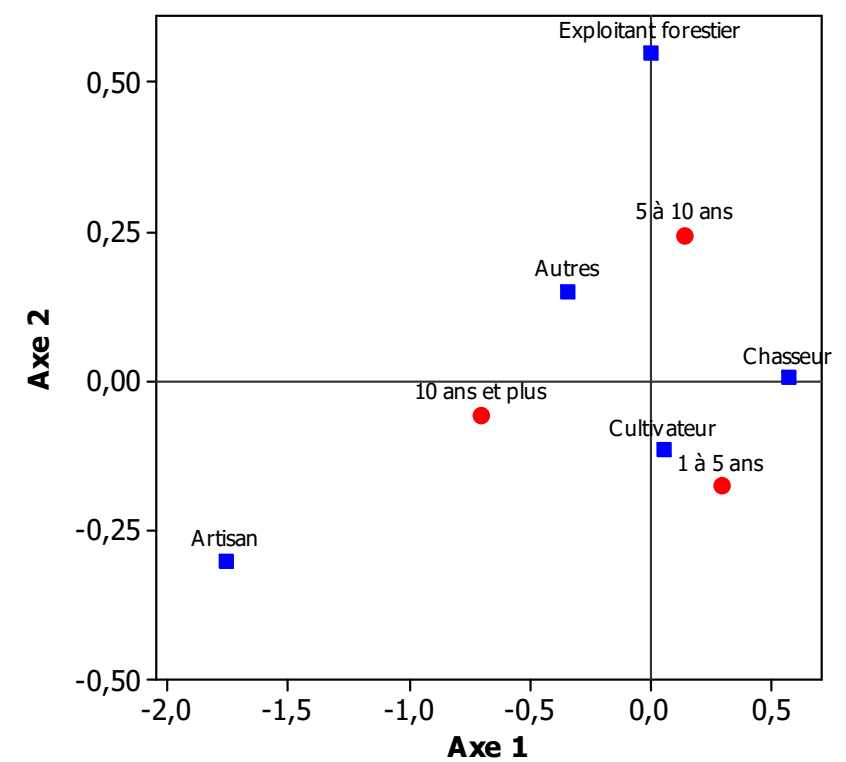

Figure 5. Projection des perceptions de la durée de fragmentation des forêts denses et des différents groupes socioprofessionnels dans un système d'axes

Sur l'axe 1, les artisans et les autres acteurs estiment que la durée de la fragmentation des forêts denses est de 10 ans et plus alors que les chasseurs pensent que cette durée est comprise entre 1 et 5 ans. Sur l'axe 2, les

\section{DISCUSSION}

Facteurs déterminants de la fragmentation des forêts denses : Les facteurs déterminants de la fragmentation des forêts denses n'épargnent pas aussi les forêts sacrées. En effet, les forêts sacrées sont des exemples de traditions locales qui contribuent au sauvetage de la flore et de la faune menacées de disparition (Camara, 1994 ; Chandrashekara, Sankar, 1998 ; Kokou et al., 1999). Plusieurs auteurs ont évoqué la nécessité de tirer certaines leçons de ces systèmes de gestion locale (Ostrom, 1997 ; Ramakrishnan et al., 1998). Mais, actuellement, plusieurs éléments concourent à leur déclin, notamment la croissance démographique, les défrichements et les feux de végétation, l'expansion incontrôlée des habitations, l'érosion des croyances religieuses traditionnelles et la faiblesse du pouvoir des chefs religieux. Selon Bamba (2010) aussi bien dans le Bas-Congo que dans la Province orientale de la République Démocratique du Congo, la déforestation est le plus souvent liée à trois facteurs principaux que sont l'exploitation et la gestion non rationnelle des ressources forestières, la pression démographique et le faible niveau de vie dû à la paupérisation croissante de la population. Selon I'UICN/PACO (2008), les aires protégées de la cultivateurs pensent que la durée de la fragmentation des forêts denses est de 1 à 5 ans alors que les exploitants forestiers l'estiment entre 5 et 10 ans.

Guinée sont soumises à de fortes pressions, telles que l'agriculture, le pastoralisme, les feux de végétation, la pêche, les activités minières, le prélèvement des produits forestiers non ligneux, les conflits population/parc (dommages aux cultures et bétail/faune). Ainsi, dans le Badiar, l'exploitation du bois est le fait d'exploitants clandestins, de fabricants de fours à brique et charbon, de scieurs traditionnels. De même, dans le BafingFalémé, il y a une exploitation excessive du bois d'œuvre car malgré la délivrance des droits de coupe par l'administration forestière, l'exploitation va trop souvent au-delà de ces permis, et l'exploitation clandestine (anarchique) s'y ajoute. La généralisation des véhicules a entraîné une augmentation de l'exploitation, que ce soit de caillcédrat ou de lengué. Le vène est de plus en plus attaqué car il a une forte valeur et les autres espèces disponibles auparavant se raréfient. Dans le Haut Niger, les responsables du déclenchement des feux sont les chasseurs, les éleveurs, les récolteurs de miel, les agriculteurs et les pêcheurs (fumage de poissons). Des comités villageois de gestion des feux ont été mis en place, des réunions de sensibilisation sont organisées. La collaboration avec les services techniques, les médias et 
les ONG ont permis des mises à feu précoce et la réalisation de pare-feux. De même, dans le Badiar, les causes des feux sont multiples : récolteurs de miel, chasseurs, pêcheurs, fours à briques, carbonisation, agriculteurs, récolteurs de vin de rônier. La sensibilisation et la collaboration avec les services techniques aboutit à des mises à feux précoces en novembre-décembre et en janvier-février par les agents de faune, et à des feux précoces autour des villages. Ce constat fait par l'UICN dans les aires protégées de la Guinée ne fait donc que confirmer nos résultats obtenus sur les facteurs déterminants de dégradation des forêts denses des Monts Kouffé au Bénin. Mais actuellement et habituellement, les feux allumés par l'Homme et qui parcourent les savanes périphériques ne pénètrent pas à travers les lisières de la forêt (Swaine, 1992). Ils sont en particulier arrêtés par la végétation basse et luxuriante (lianes, fougères, arbustes) qui matérialisent les lisières et qui restent vertes durant la saison sèche (Spichiger et Parnard, 1973). Toutefois durant certaines années nettement plus sèches que la normale, les feux réussissent à pénètrer dans les forêts semi décidues périphériques. Au Ghana, Hall et Swaine (1981) et Swaine (1992) ont ainsi défini dans la forêt au contact des savanes une "zone du feu » où le feu pénètrerait environ tous les 15 ans. De nombreux témoignages rapportent qu'au début de 1983, année exceptionnellement sèche causée par un allongement de presque 2 mois de la saison sèche annuelle, une propagation exceptionnelle des feux en forêt est intervenue en Côte d'Ivoire (Berrault, 1990), au Ghana (Swaine, 1992; Hawthorne, 1993), mais aussi au Cameroun, en Centrafrique, au Zaïre et probablement dans d'autres régions forestières. A Bornéo il en a été de même cette année là durant laquelle des feux ont pu même se propager à travers des forêts humides sempervirentes. Cette sécheresse à Bornéo a été

\section{CONCLUSION}

Grâce à cette étude basée sur les perceptions des populations locales, les facteurs déterminants de la fragmentation des forêts denses de la région des Monts Kouffé sont connus. Ceux-ci ont pour noms ; exploitation forestière, agriculture, feux de végétation, érosion hydrique, pauvreté des sols. Les résultats de l'analyse factorielle de correspondance (AFC) entre les facteurs de la fragmentation des forêts denses ont révélé que sur les deux premiers axes le pourcentage des informations obtenues n'est pas le même au niveau des groupes socioculturels $(98,94 \%)$ qu'à celui des catégories associée au développement d'un El Nino très intense (fort réchauffement des eaux marines de surface) (Goldammer et Seiberr, 1990). La propagation des feux dans l'intérieur du bloc forestier est donc un phénomène très inhabituel et il en est de même pour les feux naturels déclenchés en forêt par la foudre, qui restent circonscrits. On peut citer l'exemple d'un Pterocarpus soyauxii, haut de $40 \mathrm{~m}$, qui a brulé dans une forêt du centre du Gabon (Turin et al., 1994). Toutefois, lorsqu'exceptionnellement le feu dévaste un secteur forestier, si aucun autre feu ne survient dans les mois ou les années suivantes (Swaine, 1992), la régénération forestière est alors très rapide et elle débute par un faciès particulier où dominent des grandes Monocotylédones appartenant aux Marantaceae et Zingiberaceae et dans lesquelles la densité des arbres de type pionnier est faible car ils sont étouffés par le développement de ces grandes herbacées (De Foresta, 1990).

Ordre d'importance des facteurs déterminants de la fragmentation des forêts denses: Les résultats de l'analyse factorielle de correspondance (AFC) entre les facteurs de fragmentation des forêts denses ont révélé que sur les deux premiers axes, le pourcentage des informations obtenues n'est pas le même au niveau des groupes socioculturels et des catégories socioprofessionnelles. Chez les groupes socioculturels, on note $98,94 \%$ tandis que chez les catégories socioprofessionnelles la valeur est de $79,10 \%$. Cette différence de pourcentage de $19,84 \%$ (20\% environ) traduit probablement le degré d'expérience empirique élevé des groupes socioculturels par rapport à celui des catégories socioprofessionnelles. Car les premiers auraient vécus dans le temps et l'espace et, vivent encore les effets néfastes de chacun des différents facteurs déterminants de la fragmentation des forêts denses dans leur environnement.

socioprofessionnelles $(79,10 \%)$. Pour les groupes socioculturels fon et Bariba, l'érosion hydrique est le principal facteur de la fragmentation des forêts denses. Tandis que pour les Lokpa et les Peulh la fragmentation des forêts denses a pour cause la pauvreté des sols. De même, les groupes socioculturels estiment que la durée de la fragmentation des forêts denses est de 1 et 5 ans ; 5 et 10 ans pour certains et de 10 ans et plus pour d'autres. Alors que pour les catégories socioprofessionnelles, cette durée est de 1 à 5 ans et, de 5 à 10 ans seulement. Les résultats ainsi obtenus de 
cette étude permettront aux ONG et chercheurs du domaine de la conservation des ressources naturelles, d'alerter les décideurs politiques surtout les Députés pour

\section{REMERCIEMENTS}

Nous remercions très sincèrement les autorités locales et les groupes socioculturels et catégories socioprofessionnelles des villages riverains de la forêt classée des Monts Kouffé. Car ceux-ci n'ont ménagé aucun effort pour nous assister, nous écouter et nous

\section{RÉFÉRENCES BIBLIOGRAPHIQUES}

Azalou, 2008. Méthodologie de la recherche en sciences de l'homme et de la santé Notes de cours en D.E.A et D.E.S.S, Gestion de l'Environnement. FLASH, UAC, Bénin.

Bamba I., 2010. Anthropisation et dynamique spatiotemporelle de paysages forestiers en République Démocratique du Congo. Thèse de Doctorat. Service d'Écologie du Paysage et Systèmes de Production Végétale. Faculté des Sciences. Université Libre de Bruxelles. Belgique.181 $\mathrm{p}$.

Berrault JG, 1990. Comparaison d'écosystèmes forestiers naturels et modifiés après incendie en Côte d'Ivoire. In H. Puig (ed.) Atelier sur l'Aménagement et la Conservation de l'Ecosystème Forestier tropical humide, Cayenne, pp. 1-25 (Paris : UNESCO).

Camara T, 1994. Biodiversité et forêts sacrées en Casamance, région de Ziguinchor. Afrinet Report 10, Unesco-Rosta, Dakar, Sénégal, $65 \mathrm{p}$.

Chandrashekara UM, Sankar S, 1998. Ecology and management of sacred groves in Kerela, India. Forest Ecology and Management, 112, p.162177.

De Foresta H, 1990. Origine et évolution des savanes intramayombiennes (Congo). II. Apports de la botanique forestière. In Lanfranchi, R. et Schwartz, D. (eds.), Paysages Quaternaires de l'Afrique Centrale Atlantique, pp. 326-335 (Paris : ORSTOM).

Foody G.M., 2002, "Status of land cover classification accuracy assessment", Remote Sensing of Environment, vol. 80, p. 185-201.- Goldammer JG et Seiberr B 1990. The impact of droughts and forest fires on tropical lowland rain forest of East Kalimatan. In Goldammer, J.G. (ed.), Fire in the Tropical Bioto. Ecological Studies, 84 (Berlin: Springer-Verlag). qu'ils prennent des lois à l'Assemblée Nationale en vue d'arrêter et de corriger cette situation.

fournir des informations utiles lors de nos travaux de terrain. Nous n'oublions pas aussi tous ceux qui de près ou de loin ont efficacement contribué scientifiquement à ce travail.

Hall JB et Swaine MD 1981. Distribution and ecology of vascular plants in a tropical rain forest: Forest vegetation in Ghana (La Haye: W. Junk Publ.).

Hawthorne WD, 1993. Fire damage and forest regeneration in Ghana, Ghana Forestry Dept. Report (Londres: ODA et NRI).

Hien M, Boussim J, Sita G, 2002. L'Utilisation de Burkea Africana Hook (Caesalpiniaceae) par les populations d'éléphants (Loxodonta Africana Blumen Bach) dans le Ranch de Gibier de Nazinga (Burkina Faso). Ann Bot Afr Ouest 02 : 25-35.

Kokou K, Afiademanyo K, Akpagana K, 1999. Les forêts sacrées littorales du Togo : rôle culturel et de conservation de la biodiversité. J. Rech. Sci. Univ. Bénin (Togo), 3 (2) : 91-104.

Maley J,.Fluctuations majeures de la forêt dense humide africaine au cours des vingt derniers millénaires.Chapitre 3 : contribution 95-071 de l'institut des sciences de l'évolution de montpellier (ISEM/CNRS, URA 327), pp.55-76.

Site internet: http://horizon.documentation.ird.fr/exldoc/pleins_textes/divers09-03/010009722.pdf (consulté le 18/12/ 12 à 3h00').

Mercier J-R., 1991. La déforestation en Afrique. Situation et perspectives, Paris, Edisud.

Ostrom E., 1997. Local institutions for resource management. In: Beyond fences: seeking social sustainability in conservation. Volume 2. BorriniFeyerabend G. (ed). IUCN, Gland, Suisse, p. 14-16.

Ramakrishnan PS, Saxena KG, Chandrashnan UM, 1998. Conserving the sacred: for biodiversity management. UNESCO, Oxford IBH Publ., New Delhi, India, $480 \mathrm{p}$.

Sinsin AB, 1996. Aménagement des forêts classées de Wari-Maro, des Monts Kouffé et d'Agoua Volet 
Aménagement de la MDR/DFRN/BPF Cotonou République du Bénin. Janvier. $43 \mathrm{p}$.

UICN/PACO, 2008. Evaluation de l'efficacité de la gestion des aires protégées : aires protégées de la République de Guinée. 55 p.

Spichiger R et Parnard C, 1973. Recherches sur le contact forêt-savane en Côte d'Ivoire : Etude de recrû forestier sur des parcelles cultivées en lisière d'un îlot forestier dans le sud du pays baoulé. Candollea. 28, 21-37.

Swaine MD, 1992. Characteristics of dry forest in West Africa and the influence of fire. J. vegetation Sc., 3, 365-374.

Turin CE, White LJ, Mackanga-Missandzou A et Fernandez-Puente M, 1994. Coup de foudre fatal pour un seigneur de la forêt : observation d'un phénomène naturel au cœur de la Réserve de la Lopé au Gabon. Canopée, Bull. ECOFAC, 1,9 . 